\title{
Measuring neighborhood deprivation for childhood health and development - scale implications in rural and urban context
}

\author{
Alexandra Ursache, ${ }^{1}$ Seann Regan, ${ }^{2}$ Allison De Marco, ${ }^{3}$ Dustin T. Duncan, ${ }^{2}$ and The Family Life \\ Project Key Investigators
}

${ }^{1}$ Department of Population Health, NYU Grossman School of Medicine, New York, NY;

${ }^{2}$ Department of Epidemiology, Columbia University Mailman School of Public Health, New York, NY;

${ }^{3}$ Frank Porter Graham Child Development Institute, UNC at Chapel Hill, Chapel Hill, NC, USA

\begin{abstract}
Neighborhood deprivation plays an important role in childhood health and development, but defining the appropriate neighborhood definition presents theoretical as well as practical challenges. Few studies have compared neighborhood definitions outside of highly urbanized settings. The purpose of the current study was to evaluate how various administrative and ego-centric neighborhood definitions may impact measured exposure to deprivation across the urban-rural continuum. We do so using the Family Life Project, a prospective longitudinal population-based sample of families living in North Carolina and Pennsylvania (USA), which also sets the stage for future investigations of neighborhood
\end{abstract}

Correspondence: Alexandra Ursache, Department of Population Health, NYU Grossman School of Medicine, 227 East 30th Street, New York, 227 East 30th St, 7th Floor, New York, NY 10016, USA. E-mail: alexandra.ursache@nyumc.org

Key words: Rural; neighborhood deprivation; neighborhood definition.

Acknowledgements: we would like to express our deep gratitude to the families who participated in the study and the data collectors who worked on this study. This research is based on data from the study entitled The Family Life Project originally supported by National Institute of Child Health and Human Development Grants P01 HD39667-01A1, P01 HD039667-06, with co-funding from National Institute on Drug Abuse and the Center for Minority Health. This work was supported by the National Heart, Lung, and Blood Institute grant K01HL138114 to the first author. Funding sources were not involved in the study design or in the decision to submit the article for publication. The content is solely the responsibility of the authors and does not necessarily represent the official views of the funders.

Received for publication: 30 July 2020.

Revision received: 6 November 2020.

Accepted for publication: 15 November 2020.

CCopyright: the Author(s), 2021

Licensee PAGEPress, Italy

Geospatial Health 2021; 16:926

doi:10.4081/gh.2021.926

This article is distributed under the terms of the Creative Commons Attribution Noncommercial License (CC BY-NC 4.0) which permits any noncommercial use, distribution, and reproduction in any medium, provided the original author(s) and source are credited. impacts on childhood health and development. To measure neighborhood deprivation, a standardized index of socioeconomic deprivation was calculated using data from the 2007-2011 American Community Survey. Families' residential addresses when children were 2 months of age $(n=1036)$ were geocoded and overlaid onto a deprivation index layer created at the census block group level to construct multiple administrative and ego-centric neighborhood definitions. Friedman tests were used to compare distributions of neighborhood deprivation across these neighborhood definitions within urbanized areas, urban clusters, and rural areas. Results indicated differences in urbanized areas (Chisquare $=897.75, \mathrm{P}<0.001)$ and urban clusters (Chi-square $=687.83$, $\mathrm{P}<0.001$ ), but not in rural areas (Chi-square $=13.52, \mathrm{P}=0.332$ ). Findings imply that in urban areas, choice of neighborhood definition impacts measured exposure to neighborhood deprivation. Although exposure to neighborhood deprivation appears to be less sensitive to neighborhood definition in rural areas, researchers should apply theoretical reasoning to choose appropriate definitions of children's neighborhood.

\section{Introduction}

Understanding the role of neighborhood context is an important goal across multiple facets of early childhood research including the investigation of health and developmental outcomes as well as early care and education (Minh et al., 2017; Leventhal, 2018; Lin and Madill, 2019). Neighborhood socioeconomic conditions have been shown to be an important facet of the neighborhood context in childhood that predicts achievement, socioemotional, and behavioral outcomes (Leventhal, 2018). In a recent scoping review of 42 studies examining neighborhood effects on child development, 34 studies examined some measure of neighborhood socioeconomic status such as neighborhood poverty and the majority found that higher neighborhood poverty or deprivation was related to poorer developmental outcomes (Minh et al., 2017).

Defining the appropriate neighborhood definition that correctly aligns with the phenomenon being studied presents theoretical as well as practical challenges, including that neighborhoods have been conceptualized in a variety of ways from distinct geographic spaces, to communities of individuals. Researchers often use the best available data, or data at census geographies or administrative units that are easily accessible and make the assumption that the data represented at that geography correspond to what they are attempting to measure (Fowler and Jensen, 2020). Within the field of child development, most studies relating neighborhood factors 
to early childhood development have also relied solely on administrative boundaries, primarily census boundaries (e.g., census tracts), to define neighborhoods (Minh et al., 2017), similar to the broader literature on neighborhoods and health (Duncan et al., 2018). Imprecise and atheoretical definitions of neighborhood contexts are problematic, however; they are problematic not only because they may lead to inaccurate descriptions of the contexts in which children are living, known as spatial misclassification, but also because they lead to conflicting findings regarding relations between children's neighborhood context and their health and developmental outcomes (Duncan et al., 2014). While these challenges are not the specific focus of this study, they have been discussed elsewhere in detail (De Marco \& De Marco, 2010; Duncan et al., 2018). Briefly, the modifiable areal unit problem (MAUP) describes how the estimate of exposure based on spatial units (in this case neighborhood) changes based on the zone and size of the areal definition. For example, when census tracts are used as the definition of neighborhood, the measurement of exposures may be less accurate for an individual who lives on the border of that tract than for an individual who lives in the center of that tract. The MAUP may also impact correlations between variables derived from an improperly defined scale, as the scale used increases misinterpretations of correlation between variables may occur. Edge effects also highlight how people can travel across the defined borders of a neighborhood which may lead to distortion in their measured exposure to a certain aspect of the neighborhood (Ripley, 1981; Fortney et al., 2000). A similar but distinct spatial phenomenon, the uncertain geographic context problem, describes the uncertainty around which geographic contexts may actually influence individuals' health and development (Kwan, 2013). To illustrate, in trying to estimate the effect of a child's neighborhood on their development of academic skills, there is uncertainty as to whether examining characteristics of the home neighborhood would be most important or whether investigating exposures in the school neighborhood would be most important. Furthermore, individuals may spend significant time outside of their residential neighborhoods and the characteristics of these activity spaces may greatly differ from those of their residential neighborhoods (Zenk et al., 2011).

It is important to note that the unit of analysis, and selection criteria for what constitute a neighborhood often have strengths and weaknesses. Administrative units are geographically bounded areas that are explicitly delineated by local, state, or federal government entities, making these units policy-relevant. While administrative units may not be the most representative of areas in which individuals spend time and move throughout their day (as highlighted by spatial misclassification bias), they are widely used, and also represent geographies in which public funds are allocated. Egocentric buffers created around geocoded addresses offer a more individually targeted neighborhood definition that may more closely align to the individual lived experience; again the full breadth of this has been discussed in more depth elsewhere (Duncan et al., 2018).

Despite the importance of considering the role of the chosen neighborhood definition, few studies have investigated the extent to which measured exposure to deprivation is dependent upon the neighborhood definition that is chosen. Among the studies that have investigated this issue, to our best knowledge all have relied solely on comparing various administrative boundaries and have been conducted in urban settings (Schuurman et al., 2007; Dumedah et al., 2008; Cabrera-Barona et al., 2016). A handful of urban-based studies have examined the ways in which various neighborhood exposures (e.g., tobacco outlets) differ across both egocentric and administrative neighborhood definitions (Duncan et al., 2014; Vallée \& Shareck, 2014; Östh et al., 2015; Sluiter et al., 2015; Perchoux et al., 2016). However, almost $20 \%$ of the United States population, including over 13 million children, live in rural areas.

Few studies have examined neighborhoods in rural areas and much more work is needed to understand the ways in which neighborhood definition is related to measured exposure to neighborhood deprivation in these non-urban areas (De Marco \& De Marco, 2010). Non-urban areas present unique geographies that require distinct considerations. Administratively defined neighborhoods such as census tracts are geographically larger in rural areas which makes it more difficult to assume homogeneity within the unit (De Marco \& De Marco, 2010). Heterogeneity within the neighborhood unit can limit variation between neighborhoods and thus make it more difficult to detect neighborhood effects (O'Campo, 2003; De Marco and De Marco, 2010; Duncan et al., 2018). Furthermore, distances between neighbors and distances to basic amenities may be larger in rural than in urban areas, necessitating that ego-centric buffers be larger than have been traditionally used in urban research. Alternatively, communities may be organized around a central institution such as a church or a school, creating a strong sense of social cohesion and a neighborhood definition based on social ties rather than on an administratively defined geographic place (De Marco and De Marco, 2010). Overall, there is a lack of consensus on how to best define rural neighborhoods. Further work is needed to examine how different definitions of neighborhood affect the level of contextual exposures in order to inform investigations of how neighborhood context impacts the development of children growing up in non-urban areas.

\section{The current study}

The Family Life Project (FLP) is a population-based study that provides a rich opportunity to explore how urban and rural neighborhood context relates to many aspects of childhood development and family functioning. The purpose of the current work was to evaluate how various neighborhood definitions may impact measured exposure to deprivation across the urban-rural continuum, providing a meaningful contribution to the literature. To this end we use neighborhood metrics commonly used in health research, both administrative (i.e., census tract, block group, and school district) and egocentric geographical information system (GIS) derived neighborhood definitions. For the current analysis we disaggregated egocentric buffers into two groups, Euclidean and network based. Euclidean, or straight-line distances have some strengths in that they are computationally simple, have been used in many research projects in the past (Duncan et al., 2014; Xu et al., 2014) and represent a general area in which an individual might experience the surrounding environment. However, network, or street based approaches are likely more accurate as they are calculated along physical features (the street network) that individuals can walk, bike, or drive on. In this study, we created both Euclidean and network based buffers at 0.5 mile $/ 0.8 \mathrm{~km}, 1$ mile/1. $6 \mathrm{~km}, 2$ mile $/ 3.2 \mathrm{~km}, 3$ mile $/ 4.8 \mathrm{~km}$, and 5 mile $/ 8 \mathrm{~km}$ distances around individual geocoded addresses. Wider buffers were included to account for the geographic spread of rural settings, which as we mentioned has been rarely implemented in neighborhood and health research. In line with past studies, we first examine correlations across these multiple administrative and egocen- 
tric neighborhood definitions, and we then examine distributional/rank order differences in measured exposure to neighborhood deprivation across these neighborhood scales and zones (Duncan et al., 2014). In doing so, we separately examine these differences across urbanized areas, urban clusters, and rural areas.

\section{Materials and methods}

\section{Sample}

The FLP was designed to study families in areas of high child rural poverty in two U.S. states (North Carolina and Pennsylvania). Complex sampling procedures were used to recruit a representative sample of 1292 families at the time of the target child's birth, with low-income families in both states being over-sampled and African Americans oversampled in North Carolina. [Families were designated as low-income if reported household income was less than or equal to two times the federal poverty threshold for a given household size, use of social services requiring a similar income requirement (e.g., food stamps, government programs for food provision for women and children, public health care coverage), and/or primary and secondary heads of household both of whom had less than a school education (Willoughby et al., 2013).]. Further details of FLP sampling plan and recruitment procedures are available in Vernon-Feagans et al. (2013). This study utilizes data on families' residential addresses $(n=1036)$ when children were 2 months of age. Missing data are a result of either residential location information that could not be corroborated or residential location information not being available.

\section{Measures}

\section{Neighborhood deprivation index}

There has been much discussion to date on appropriate methods for the creation of neighborhood deprivation indexes [neighborhood deprivation index (NDI)], including the benefits and shortcomings of data reduction techniques such as factor analysis and principle components analysis, versus utilizing individual variables (poverty for example) that may more directly align with policy levers. Additionally, scholars have debated how to create deprivation indexes for comparable use across studies (Messer et al., 2006; Lian et al., 2016; Wan and Su, 2016). In this study, we compute commonly used metrics of deprivation for the study area of North Carolina and Pennsylvania to set the stage for future investigations using this measure of neighborhood deprivation as a potential explanatory variable in early childhood development and family health and well-being.

A deprivation index was calculated at the census block group level. Census block groups are statistical divisions of census tracts, are generally defined to contain 600 to 3,000 people, and are the smallest geographical unit for which American Community Survey data are available. Indicators of deprivation were calculated using the American Community Survey (ACS) 5-year estimate data. The input indicators measuring neighborhood disadvantage for these analyses included: the percentage of individuals in the families' block group reporting annual income below the federal poverty level, the percentage of households that were female headed, the percentage of the population that was less than 18 years of age, the percent of the population who was over the age of 25 and had graduated high school (reverse-scored), and the percentage of the labor force that was unemployed. These measures were taken directly from commonly used neighborhood deprivation indices and specifically from prior work which has examined neighborhood deprivation in the FLP sample (Finegood et al., 2017). Because data were skewed, $\mathrm{Z}$ scores were created for each component variable. A continuous index of neighborhood disadvantage was generated by taking the $\mathrm{Z}$ scores from each of the five indicators and averaging the standardized indicators into a final index.

Some considerations should be noted when using ACS data. The ACS utilizes a rolling sample methodology so data are not from a specific year, rather they are an aggregation across a certain time frame. For example, we use 2011 ACS data, which is a 5-year average of ACS data obtained from 2007-2011. The resulting NDI will have the same potential accuracy issues and errors contained within the American Community Survey data, and these errors may be spatially autocorrelated. Full details of the sampling methods in the ACS are available from the U.S. Census (US Census Bureau, April 2009). We chose these years of data to best line up with the data from the FLP project.

\section{Neighborhood definitions}

Participant families' home addresses were geocoded and then spatially linked to a suite of areal definitions of neighborhood (Table 1). These areal definitions of neighborhood include: census block groups, census tracts, and school districts. Additionally, buffers were created around participants' geocoded addresses, using Euclidean (as the crow flies) distances, and network-based distances at 0.5 mile $/ 0.8 \mathrm{~km}, 1$ mile/ $1.6 \mathrm{~km}, 2$ mile $/ 3.2 \mathrm{~km}, 3$ mile $/ 4.8 \mathrm{~km}$, and 5 mile $/ 8 \mathrm{~km}$. These distances are commonly used in and theoretically relevant for research in spatial epidemiology (for reviews see (De Marco and De Marco, 2010; Duncan et al., 2018). For example, some distances represent walkable areas in urban settings whereas others represent the distance to obtain goods or services in a dispersed rural setting. For census block groups, the neighborhood deprivation index was a spatial join of participant geocoded address locations, so if an address fell within a block group it was assigned the neighborhood deprivation index for that block group. For census tracts, school districts, network buffers, and Euclidean buffers an area weighted average of the block group level neighborhood deprivation index was calculated, all geospatial analysis was conducted with ArcGIS version 10.8 (Beyer, 2012; Environmental Systems Research Institute (ESRI), 2019; R Core Team, 2017). The neighborhood deprivation index was built for every block group in both NC and PA and as such is relative to neighborhood deprivation levels across the two states. A full discussion of definitions of neighborhood and the spatial construction of neighborhood has been discussed elsewhere; here we aimed to align early childhood development within this body of research (De Marco and De Marco, 2010; Diez Roux and Mair, 2010; Crawford et al., 2014; Duncan et al., 2018; Hobbs et al., 2018).

\section{Urban-rural classification}

The Census Bureau classifies geographical areas into urban and rural areas with urban areas being divided into urbanized areas and urban clusters (US Census Bureau, February 2020). Urbanized areas identify densely populated urban areas of 50,000 or more people. Urban clusters are defined based on the same criteria as urbanized areas but contain at least 2500 and less than 50,000 peo- 
ple. Rural areas include all population, housing, and territory outside of urban areas.

\section{Statistical analysis}

We first examined descriptive statistics for the neighborhood deprivation index at each of the neighborhood definitions - census block groups, census tracts, school districts, Euclidean buffers, and network buffers. To examine the relative differences between neighborhood deprivation across neighborhood definitions, we calculated Spearman's correlation coefficients which are more robust when variables have a skewed distribution. Finally, we used the Friedman test to compare overall differences in neighborhood deprivation across the various neighborhood definitions. This approach follows that of previous work examining how quantification of neighborhood exposures differ across neighborhood definitions (Duncan et al., 2014). When the null hypothesis was rejected at the $\mathrm{P}<0.05$ level, Dunn-Bonferroni post-hoc tests were used to determine which neighborhood definitions led to differences in measured exposure to neighborhood deprivation. We report adjusted p-values for the post-hoc tests. Correlations and Friedman's test comparison were conducted separately for neighborhoods in urbanized areas $(n=376)$, urban clusters $(n=333)$, and rural areas $(n=327)$. All analyses were conducted in SPSS 26.

\section{Results}

Figures 1 and 2 depict boxplots showing medians and distributions of neighborhood deprivation for all children across neighborhood definitions in urbanized areas, urban clusters, and rural areas. Table 2 presents means and standard deviations of the level of neighborhood deprivation for children in each neighborhood defi-

Table 1. Neighborhood definitions and why they matter for child development.

$\begin{array}{lll}\text { Neighborhood definition } & \text { How it is defined } & \text { An } \\ \text { Census Tract - } & \text { Census tracts in the United States are designed for } & \text { The } \\ \text { administrative unit } & \text { analyzing census data temporally. Typically tracts } & \text { contain approximately 4000 people } \\ & \text { (generally they have a population size between } \\ & 2000 \text { and } 8000 \text { people). Tracts are by nature designed } \\ & \text { to be homogenous spatial units with respect to population } \\ \text { characteristics that allow researchers to track trends } & \text { through time. Tracts are small subdivisions of a county. }\end{array}$

\begin{tabular}{|c|c|c|}
\hline $\begin{array}{l}\text { Census Block Group - } \\
\text { administrative unit }\end{array}$ & $\begin{array}{l}\text { Census block group are a statistical subdivision of a census tract, } \\
\text { typically bounded by physical features such as road, and represent } \\
\text { a cluster of census blocks within the same census tract. } \\
\text { Typically, census block groups have a population of } 600 \text { to } 3000 \\
\text { people with an average population of approximately } 1000 \text { residents. }\end{array}$ & $\begin{array}{l}\text { Funding for many programs that support optimal child development } \\
\text { are allocated based on Census data, including WIC, SNAP, Head Start, } \\
\text { State Children's Health Insurance Program, and the Child Care and } \\
\text { Development Grant (Hotchkiss and Phelan, 2017). } \\
\text { Accurate Census Bureau data based on census geography enable } \\
\text { federal programs to fund initiatives by using population counts } \\
\text { and characteristics to target and distribute those funds. }\end{array}$ \\
\hline $\begin{array}{l}\text { School District - } \\
\text { administrative unit }\end{array}$ & $\begin{array}{l}\text { School Districts data use the boundaries of all school districts } \\
\text { (Elementary, Secondary, and Unified) into a single file. Data come } \\
\text { from the U.S. Department of Education's National Center for } \\
\text { Education Statistics. School Districts are geographic entities } \\
\text { and governmental units that operate schools and provide }\end{array}$ & $\begin{array}{l}\text { School attendance zones have been used in a variety of ways relevant to } \\
\text { child development research including Safe Routes to School (walkability } \\
\text { and connectivity that enable active commuting modes; Stewart, 2011) } \\
\text { and school segregation related to availability of resources (Richards, 2014). }\end{array}$ \\
\hline
\end{tabular}

The definition of a child care desert is based on the census tract: A child care desert is any census tract with more than 50 children under age 5 that contains either no child care providers or so few options that there are more than three times as many children as licensed child care slots (Malik and Hamm, 2017).

and governmental units that operate schools and provide public educational services at the local level.

The Census Bureau uses school district boundaries to develop annual estimates of children in poverty in determination for the annual allocation of Title I funding to states and school districts.

\begin{tabular}{ll}
$\begin{array}{l}\text { Euclidean Buffer } \\
0.5,1,2,3,5, \text { mile - }\end{array}$ & Euclidean Buffers were created around participant geocoded \\
egocentric & addresses, these are considered egocentric meaning they are \\
& responsive to the individual rather than based on population \\
& (Matthews, 2011). For example census tracts are population based \\
& residentially defined units and are thus characteristic of the group, \\
& rather than the individual. Egocentric approaches have garnered \\
& support recently as more accurately representing the individual \\
& lived experience. \\
\hline Network Buffer & Network Buffers were also created around participants home \\
$0.5,1,2,3,5$, mile - & geocoded addresses, but rather than straight-line distances use \\
egocentric & the available street network data (roads). This approach may better \\
& align with where participants are physically able to move throughout \\
& the environment, although both Euclidean and network-based \\
& approaches have strengths.
\end{tabular}

Euclidean buffers have been used to examine where children spend their time. These geographic definitions are able to capture various distances from home and what a child is accessing in each, for example schools, recreation areas, food outlets, and other residences (Chambers et al., 2017). It is important to note that in rural settings buffers must be larger to capture these locations.

Network buffers are used when examining walkability to amenities or access to food retailers, such as in Sadler's work looking at adolescents' access to junk food outlets (Sadler et al., 2016). 
nition. Visual inspection of the plots shows greater variation in terms of both medians and distributions across neighborhood definitions in urbanized areas and urban clusters than in rural areas. Variation in medians appears to be particularly pronounced in urbanized areas whereas variation in distributions appears particularly pronounced in urban clusters. Both medians and distributions appear more stable across neighborhood definitions in rural areas. In urbanized areas, median levels of deprivation appear to be lower when neighborhood buffers are larger and higher at smaller neighborhood geographies.

Spearman correlation coefficients of neighborhood deprivation across neighborhood definitions in urbanized areas, urban clusters, and rural areas are shown in Tables 3-5. There were moderate to strong correlations across all neighborhood definitions in urbanized areas $(r s=0.37-0.93)$, urban clusters $(r s=0.39-0.98)$, and rural areas $(r s=0.44-0.99)$. There was some indication that correlations between administrative buffers and egocentric buffers tended to be less influenced by egocentric buffer size in rural areas than in urban areas. For example, in urbanized areas, correlations comparing census tract and Euclidean 1 mile $/ 1.6 \mathrm{~km}$ buffers were $r=0.79$ whereas correlations comparing census tract and Euclidean 5 mile $/ 8 \mathrm{~km}$ buffers were $r=0.37$. In urban clusters, these correlations were $r=0.88$ and $r=0.67$. In rural areas, however, these correlations were much more similar: $r=0.78$ and $r=0.86$. The lowest, although still moderate, correlations were between the smallest geographies (i.e., census block groups and 0.5 mile $/ 0.8 \mathrm{~km}$ buffers) and school districts in rural areas $(r s=0.435-0.491)$ and in urban clusters $(r s=0.386-0.508)$. In urbanized areas, these lower correlations were similarly seen between the smallest geographies and school districts ( $r s=0.394-0.433)$, although the lowest correlations were between Euclidean 5 mile $/ 8 \mathrm{~km}$ buffers and census geographies $(r s=0.366-0.387)$.

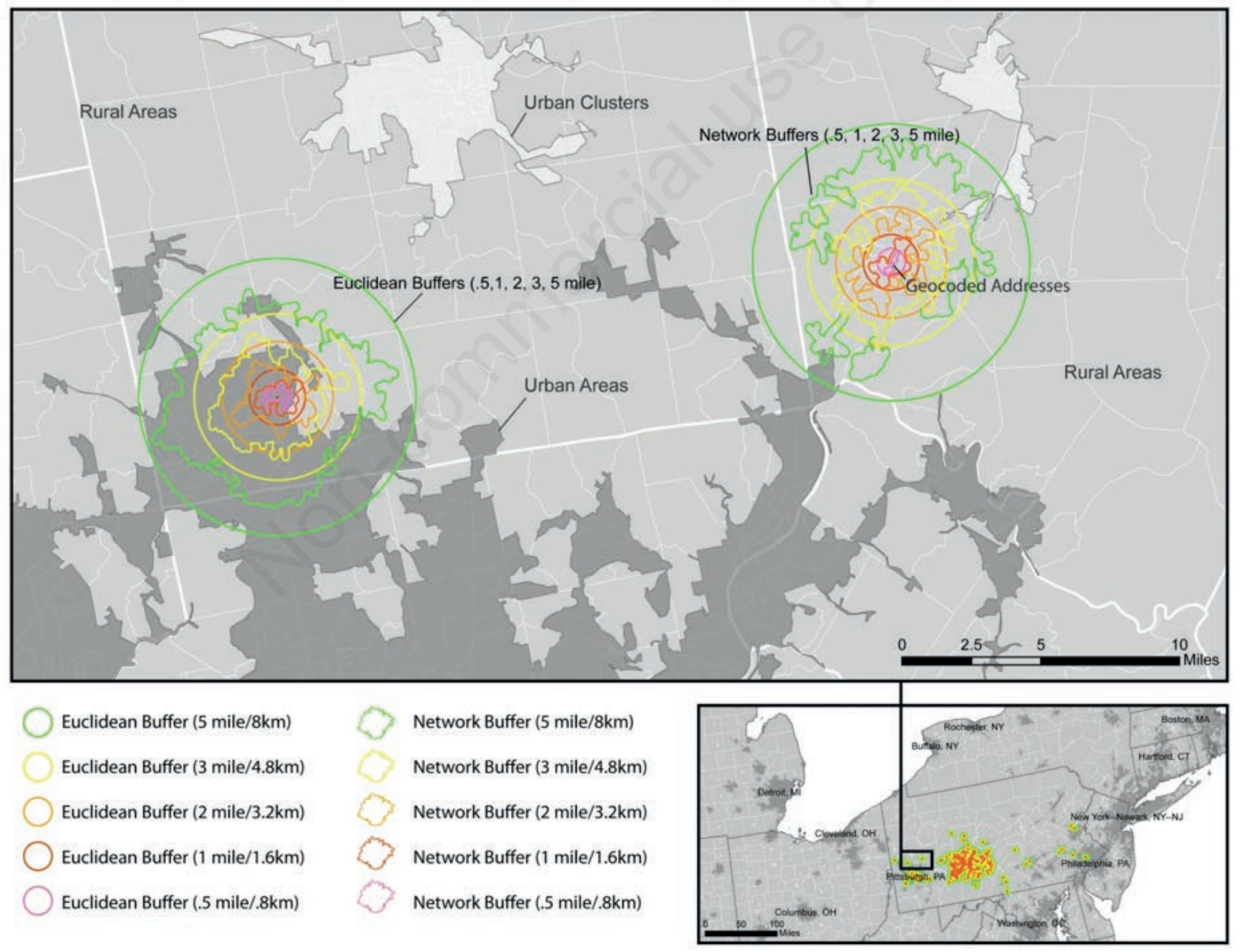

Figure 1. Map depicting an example of urbanized areas, urban clusters, and rural areas. An example of Euclidean and network buffers are shown overlaid in urbanized areas and rural areas. Network buffers are more similar to Euclidean buffers in urbanized areas than in rural areas. Note: Participant address information not visualized in map. 
Results of the Friedman tests indicated that overall, distributions of neighborhood deprivation differed across the various neighborhood definitions in urbanized areas (Chi-square $=897.75$, $\mathrm{P}<0.001$ ) and urban clusters (Chi-square $=687.83, \mathrm{P}<0.001$ ), but not in rural areas (Chi-square $=13.52, \mathrm{P}=0.332$ ). Pairwise comparisons of neighborhood definitions in urbanized areas and urban clusters (Table 6) demonstrate that there were significant differences in deprivation between most but not all neighborhood definitions. For example, in urbanized areas, census tract and Euclidean 1 mile/1.6 km buffers resulted in similar levels of exposure to neighborhood deprivation $(\mathrm{P}=1.000)$ whereas comparing census tract and Euclidean 5 mile $/ 8 \mathrm{~km}$ buffers resulted in different levels of exposure to neighborhood deprivation $(\mathrm{P}<0.001)$. Similarly, in urban clusters, census tract and Euclidean 1 mile buffers resulted in similar levels of exposure to neighborhood deprivation $(\mathrm{P}=0.109)$ whereas comparing census tract and Euclidean 5 mile $/ 8 \mathrm{~km}$ buffers resulted in different levels of exposure to neighborhood deprivation $(\mathrm{P}<0.001)$.

\section{Discussion}

While much research has sought to tease out the influence of neighborhood on various health and developmental outcomes, often researchers take an off the shelf approach to data using what datasets are available to them. This ad hoc (and often theoretically devoid) approach is problematic as it may lead to results that are both inconclusive, and that may confound findings as the measured exposure to neighborhood factors can change based on the zone
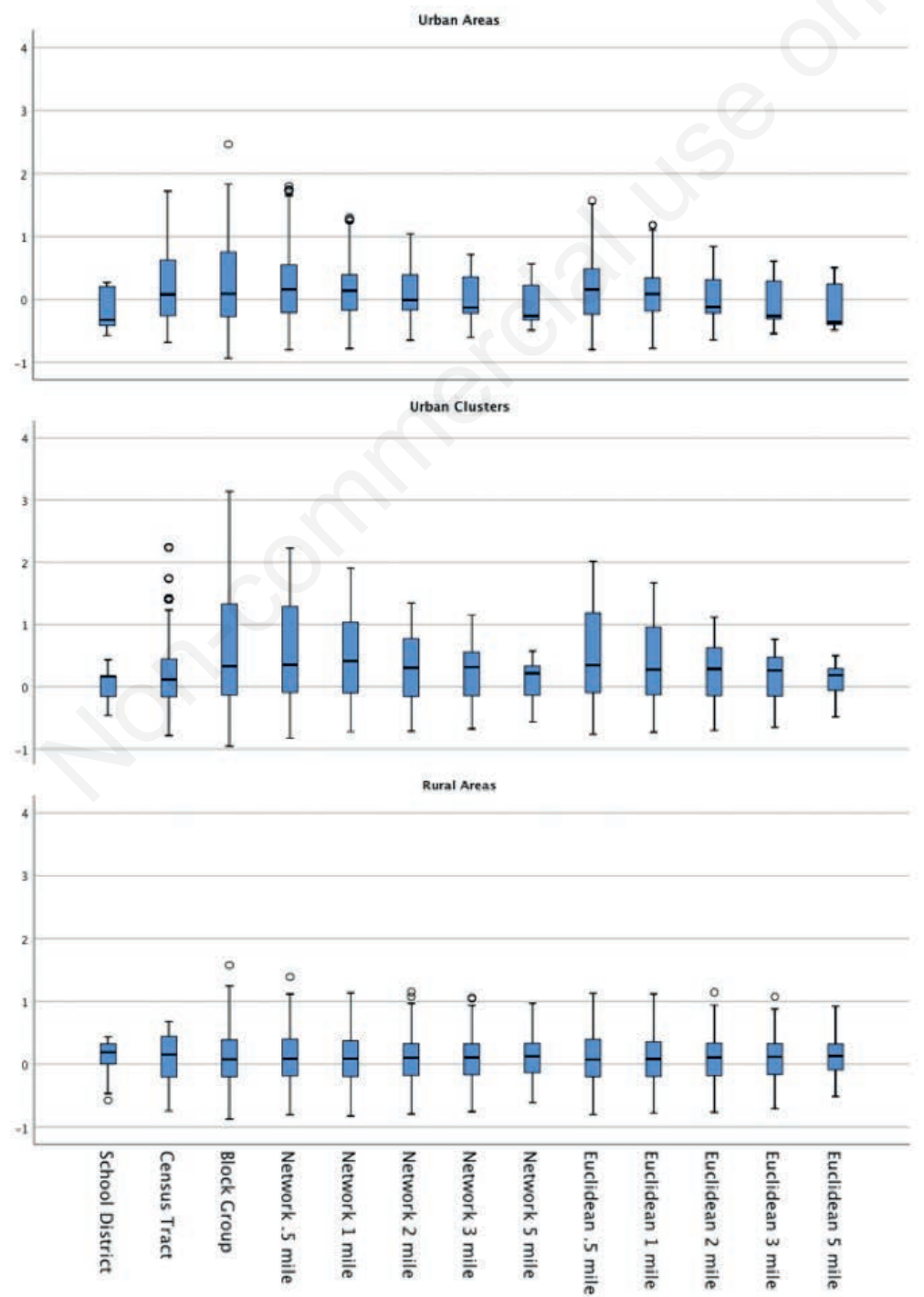

Figure 2. Boxplots depicting medians and distributions of neighborhood deprivation for each neighborhood definition in urbanized areas, urban clusters, and rural areas. 
Table 2. Descriptive statistics for neighborhood deprivation.

\begin{tabular}{lccccccccc} 
& \multicolumn{3}{c}{ Urbanived areas } & \multicolumn{3}{c}{ Urban clusters } & \multicolumn{2}{c}{ Rural areas } \\
& Mean & SD & Range & Mean & SD & Range & Mean & SD & Range \\
School District & -0.16 & 0.30 & {$[-0.57-0.27]$} & 0.05 & 0.24 & {$[-0.46-0.44]$} & 0.12 & 0.28 & {$[-0.57-0.44]$} \\
Census Tract & 0.18 & 0.54 & {$[-0.69-1.72]$} & 0.34 & 0.71 & {$[-0.78-2.23]$} & 0.11 & 0.35 & {$[-0.74-0.68]$} \\
\hline Census Block Group & 0.29 & 0.74 & {$[-0.94-2.46]$} & 0.59 & 0.94 & {$[-0.96-3.13]$} & 0.12 & 0.44 & {$[-0.87-1.57]$} \\
Network 1/2 mile & 0.23 & 0.57 & {$[-0.81-1.8]$} & 0.57 & 0.83 & {$[-0.82-2.23]$} & 0.12 & 0.41 & {$[-0.8-1.39]$} \\
\hline Network 1 mile & 0.14 & 0.43 & {$[-0.78-1.29]$} & 0.48 & 0.69 & {$[-0.72-1.9]$} & 0.11 & 0.39 & {$[-0.82-1.14]$} \\
Network 2 mile & 0.07 & 0.37 & {$[-0.65-1.04]$} & 0.35 & 0.55 & {$[-0.71-1.35]$} & 0.09 & 0.36 & {$[-0.79-1.15]$} \\
\hline Network 3 mile & -0.01 & 0.33 & {$[-0.6-0.71]$} & 0.26 & 0.43 & {$[-0.68-1.15]$} & 0.09 & 0.34 & {$[-0.75-1.06]$} \\
Network 5 mile & -0.10 & 0.30 & {$[-0.49-0.57]$} & 0.12 & 0.29 & {$[-0.56-0.58]$} & 0.10 & 0.32 & {$[-0.61-0.97]$} \\
\hline Euclidean 1/2 mile & 0.20 & 0.52 & {$[-0.8-1.57]$} & 0.52 & 0.78 & {$[-0.76-2.02]$} & 0.11 & 0.40 & {$[-0.8-1.13]$} \\
Euclidean 1 mile & 0.11 & 0.41 & {$[-0.78-1.18]$} & 0.40 & 0.64 & {$[-0.73-1.67]$} & 0.10 & 0.38 & {$[-0.77-1.12]$} \\
\hline Euclidean 2 mile & 0.00 & 0.35 & {$[-0.64-0.84]$} & 0.27 & 0.47 & {$[-0.7-1.12]$} & 0.09 & 0.35 & {$[-0.76-1.14]$} \\
Euclidean 3 mile & -0.07 & 0.33 & {$[-0.54-0.61]$} & 0.18 & 0.36 & {$[-0.65-0.77]$} & 0.09 & 0.32 & {$[-0.7-1.08]$} \\
\hline Euclidean 5 mile & -0.13 & 0.33 & {$[-0.49-0.5]$} & 0.08 & 0.27 & {$[-0.48-0.5]$} & 0.09 & 0.30 & {$[-0.51-0.92]$} \\
\hline
\end{tabular}

Note: Network and Euclidean buffer sizes are 0.5 mile/ 0.8 km, 1 mile/1.6 km, 2 mile/3.2 km, 3 mile/4.8 km, and 5 mile/8 km. SD, standard deviation.

Table 3. Correlations among exposure to neighborhood deprivation measured at various neighborhood definitions in urbanized areas.

\begin{tabular}{|c|c|c|c|c|c|c|c|c|c|c|c|c|}
\hline & 1 & 2 & 3 & 4 & 5 & 6 & 7 & 8 & 9 & 10 & 11 & 12 \\
\hline \multicolumn{13}{|l|}{ 1. School District } \\
\hline 2. Census Tract & $0.424 * *$ & & & & & & & & & & & \\
\hline 3. Census Block Group & $0.394 * *$ & $0.821^{* *}$ & & & 0 & 8 & & & & & & \\
\hline 4. Network $1 / 2$ mile & $0.419 * *$ & $0.844^{* *}$ & $0.917^{* *}$ & & $\theta^{2}$ & & & & & & & \\
\hline 5. Network 1 mile & $0.488 * *$ & $0.825^{* *}$ & $0.819 * *$ & $0.931^{* *}$ & & & & & & & & \\
\hline 6. Network 2 mile & $0.504^{* *}$ & $0.719 * *$ & $0.717^{* *}$ & $0.827^{* *}$ & $0.932 * *$ & & & & & & & \\
\hline 7. Network 3 mile & $0.641^{* *}$ & $0.584^{* *}$ & $0.614 * *$ & $0.692 * *$ & $0.778 * *$ & $0.871^{* *}$ & & & & & & \\
\hline 8. Network 5 mile & $0.794^{* *}$ & $0.456^{* *}$ & $0.469 * *$ & $0.519 * *$ & $0.610^{* *}$ & $0.665^{* *}$ & $0.815^{* *}$ & & & & & \\
\hline 9. Euclidean $1 / 2$ mile & $0.433 * *$ & $0.840 * *$ & $0.883^{* *}$ & $0.977^{* *}$ & $0.958 * *$ & $0.859 * *$ & $0.722 * *$ & $0.544^{* *}$ & & & & \\
\hline 10. Euclidean 1 mile & $0.487^{* *}$ & $0.790 * *$ & $0.780 * *$ & $0.888^{* *}$ & $0.976^{* *}$ & $0.944^{* *}$ & $0.824^{* *}$ & $0.621^{* *}$ & $0.933^{* *}$ & & & \\
\hline 11. Euclidean 2 mile & $0.644^{* *}$ & $0.622 * *$ & $0.645^{* *}$ & $0.737^{* *}$ & $0.825^{* *}$ & $0.906^{* *}$ & $0.971^{* *}$ & $0.797^{* *}$ & $0.766 * *$ & $0.867^{* *}$ & & \\
\hline 12. Euclidean 3 mile & $0.763^{* *}$ & $0.543^{* *}$ & $0.561^{* *}$ & $0.616^{* *}$ & $0.694^{* *}$ & $0.755^{* *}$ & $0.921^{* *}$ & $0.910 * *$ & $0.643^{* *}$ & $0.736^{* *}$ & $0.904^{* *}$ & \\
\hline 13. Euclidean 5 mile & $0.853^{* *}$ & $0.366^{* *}$ & $0.387^{* *}$ & $0.414^{* *}$ & $0.487^{* *}$ & $0.553^{* *}$ & $0.730^{* *}$ & $0.910^{* *}$ & $0.428 * *$ & $0.504^{* *}$ & $0.715^{* *}$ & $0.841^{* *}$ \\
\hline
\end{tabular}

Table 4. Correlations among exposure to neighborhood deprivation measured at various neighborhood definitions in urban clusters.

\begin{tabular}{|c|c|c|c|c|c|c|c|c|c|c|c|c|}
\hline & 1 & 2 & 3 & 4 & 5 & 6 & 7 & 8 & 9 & 10 & 11 & 12 \\
\hline \multicolumn{13}{|l|}{ 1. School District } \\
\hline 2. Census Tract & $0.565^{* *}$ & & & & & & & & & & & \\
\hline 3. Census Block Group & $0.386 * *$ & $0.720^{* *}$ & & & & & & & & & & \\
\hline 4. Network $1 / 2$ mile & $0.463^{* *}$ & $0.776^{* *}$ & $0.933^{* *}$ & & & & & & & & & \\
\hline 5. Network 1 mile & $0.533^{* *}$ & $0.848^{* *}$ & $0.866^{* *}$ & $0.949 * *$ & & & & & & & & \\
\hline 6. Network 2 mile & $0.579 * *$ & $0.852^{* *}$ & $0.776^{* *}$ & $0.844^{* *}$ & $0.945^{* *}$ & & & & & & & \\
\hline 7. Network 3 mile & $0.562^{* *}$ & $0.817^{* *}$ & $0.723^{* *}$ & $0.786^{* *}$ & $0.897^{* *}$ & $0.975^{* *}$ & & & & & & \\
\hline 8. Network 5 mile & $0.621^{* *}$ & $0.693^{* *}$ & $0.649 * *$ & $0.696^{* *}$ & $0.797^{* *}$ & $0.892^{* *}$ & $0.925^{* *}$ & & & & & \\
\hline 9. Euclidean 1/2 mile & $0.508^{* *}$ & $0.820^{* *}$ & $0.917^{* *}$ & $0.984^{* *}$ & $0.974^{* *}$ & $0.893^{* *}$ & $0.837 * *$ & $0.750^{* *}$ & & & & \\
\hline 10. Euclidean 1 mile & $0.569 * *$ & $0.875^{* *}$ & $0.844^{* *}$ & $0.922 * *$ & $0.986^{* *}$ & $0.959^{* *}$ & $0.911^{* *}$ & $0.818^{* *}$ & $0.963^{* *}$ & & & \\
\hline 11. Euclidean 2 mile & $0.589 * *$ & $0.837^{* *}$ & $0.759^{* *}$ & $0.823^{* *}$ & $0.925^{* *}$ & $0.984^{* *}$ & $0.983^{* *}$ & $0.923^{* *}$ & $0.878^{* *}$ & $0.946^{* *}$ & & \\
\hline 12. Euclidean 3 mile & $0.588^{* *}$ & $0.773^{* *}$ & $0.718^{* *}$ & $0.777^{* *}$ & $0.876^{* *}$ & $0.949 * *$ & $0.975^{* *}$ & $0.971^{* *}$ & $0.829 * *$ & $0.894 * *$ & $0.978^{* *}$ & \\
\hline 13. Euclidean 5 mile & $0.717^{* *}$ & $0.672^{* *}$ & $0.598^{* *}$ & $0.635^{* *}$ & $0.730^{* *}$ & $0.829 * *$ & $0.851^{* *}$ & $0.952^{* *}$ & $0.690^{* *}$ & $0.760^{* *}$ & $0.860 * *$ & $0.908 * *$ \\
\hline
\end{tabular}

\footnotetext{
${ }^{* *}$ Correlation is significant at the 0.01 level (2-tailed). Note: Network and Euclidean buffer sizes are 0.5 mile/ $0.8 \mathrm{~km}, 1$ mile/ $1.6 \mathrm{~km}, 2$ mile/ $3.2 \mathrm{~km}, 3 \mathrm{mile} / 4.8 \mathrm{~km}$, and $5 \mathrm{mile} / 8 \mathrm{~km}$.
} 
and size of the neighborhood definition. By examining if the measured exposure of neighborhood deprivation differed by the neighborhood definition used in the FLP this study addresses the aforementioned issues; the first within early childhood development to examine whether these differences occur across the urban to rural spectrum.

Overall, results demonstrated that in urbanized areas and urban clusters, neighborhood deprivation varied to a statistically significant degree based on the neighborhood definition used. These findings are largely consistent with the theory of the modifiable areal unit problem and past research which has shown that measurement of exposures is sensitive to the size and zone of the neighborhood definition in urban areas (Duncan et al., 2014). In measuring neighborhood deprivation specifically, this study builds on past work which found that the measurement of deprivation differs depending on the size of the administrative buffer chosen (Schuurman et al., 2007). Our post-hoc comparison results in urbanized areas are also consistent with past work which found no difference in deprivation exposure across census tracts and census block groups in Quito, Ecuador (Cabrera-Barona et al., 2016), although post-hoc comparisons did find differences in urban clusters. The current study extends this prior work by comparing both administrative and ego-centric neighborhood definitions of various size and demonstrating that different definitions lead to differences in measured exposure to neighborhood deprivation.

In rural areas, however, results suggested that measured exposure to neighborhood deprivation was not dependent on neighborhood definition. These results are consistent with visual inspection of the medians and distributions of neighborhood deprivation which appeared more stable across neighborhood definitions in rural areas. No prior studies have investigated this specific question in rural areas and few studies have compared neighborhood definitions for other types of exposures in rural areas. This finding is not entirely surprising in that administrative boundaries such as

Table 5. Correlations among exposure to neighborhood deprivation measured at various neighborhood definitions in rural areas.

\begin{tabular}{|c|c|c|c|c|c|c|c|c|c|c|c|c|}
\hline & 1 & 2 & 3 & 4 & 5 & 6 & 7 & 8 & 9 & 10 & 11 & 12 \\
\hline \multicolumn{13}{|l|}{ 1. School District } \\
\hline 2. Census Tract & $0.643^{* *}$ & & & & & & $x$ & & & & & \\
\hline 3. Census Block Group & $0.435^{* *}$ & $0.700^{* *}$ & & & & & & & & & & \\
\hline 4. Network $1 / 2$ mile & $0.472^{* *}$ & $0.741^{* *}$ & $0.940^{* *}$ & & & & & & & & & \\
\hline 5. Network 1 mile & $0.506^{* *}$ & $0.769 * *$ & $0.903^{* *}$ & $0.983^{* *}$ & & 8 & & & & & & \\
\hline 6. Network 2 mile & $0.552^{* *}$ & $0.810^{* *}$ & $0.864^{* *}$ & $0.945^{* *}$ & $0.976^{* *}$ & & & & & & & \\
\hline 7. Network 3 mile & $0.583^{* *}$ & $0.846^{* *}$ & $0.830 * *$ & $0.903 * *$ & $0.935^{* *}$ & $0.977^{* *}$ & & & & & & \\
\hline 8. Network 5 mile & $0.597^{* *}$ & $0.867^{* *}$ & $0.752^{* *}$ & $0.820^{* *}$ & $0.851^{* *}$ & $0.901^{* *}$ & $0.955^{* *}$ & & & & & \\
\hline 9. Euclidean $1 / 2$ mile & $0.491^{* *}$ & $0.748^{* *}$ & $0.939 * *$ & $0.994 * *$ & $0.985^{* *}$ & $0.952 * *$ & $0.913^{* *}$ & $0.829 * *$ & & & & \\
\hline 10. Euclidean 1 mile & $0.533^{* *}$ & $0.779^{* *}$ & $0.899 * *$ & $0.972^{* *}$ & $0.989 * *$ & $0.979 * *$ & $0.949 * *$ & $0.871^{* *}$ & $0.982^{* *}$ & & & \\
\hline 11. Euclidean 2 mile & $0.585^{* *}$ & $0.841^{* *}$ & $0.833^{* *}$ & $0.908^{* *}$ & $0.936^{* *}$ & $0.971^{* *}$ & $0.985^{* *}$ & $0.944^{* *}$ & $0.919 * *$ & $0.960^{* *}$ & & \\
\hline 12. Euclidean 3 mile & $0.585^{* *}$ & $0.869^{* *}$ & $0.763^{* *}$ & $0.838^{* *}$ & $0.869^{* *}$ & $0.917^{* *}$ & $0.965^{* *}$ & $0.980^{* *}$ & $0.848^{* *}$ & $0.892^{* *}$ & $0.971^{* *}$ & \\
\hline 13. Euclidean 5 mile & $0.593^{* *}$ & $0.856^{* *}$ & $0.662 * *$ & $0.728^{* *}$ & $0.754^{* *}$ & $0.807^{* *}$ & $0.873^{* *}$ & $0.958^{* *}$ & $0.735^{* *}$ & $0.778^{* *}$ & $0.873^{* *}$ & $0.946^{* *}$ \\
\hline
\end{tabular}

Table 6. Post-hoc pairwise comparisons from Friedman's tests in urbanized areas (bottom left) and urban clusters (top right).

\begin{tabular}{|c|c|c|c|c|c|c|c|c|c|c|c|c|c|}
\hline & 1 & 2 & 3 & 4 & 5 & 6 & 7 & 8 & 9 & 10 & 11 & 12 & 13 \\
\hline 1. School District & -- & $-1.79 * \sim$ & $-3.84 * \sim$ & $-4.32 * \sim$ & $-4.06 * \sim$ & $-2.88 * \sim$ & $-2.00 * \sim$ & -0.37 & $-3.93^{*} \sim$ & $-2.75^{*} \sim$ & $-1.42 * \sim$ & -0.5 & 0.05 \\
\hline 2. Census Tract & $-4.58 * \sim$ & -- & $-2.05^{*} \sim$ & $-2.54 * \sim$ & $-2.27 * \sim$ & $-1.09 * \sim$ & -0.22 & $1.42 * \sim$ & $-2.14^{*} \sim$ & $-0.96^{*}$ & 0.37 & $1.29 * \sim$ & $1.84 *$ \\
\hline 3. Census Block Group & $-4.79 * \sim$ & -0.21 & -- & -0.48 & -0.22 & $0.96^{*}$ & $1.84^{*} \sim$ & $3.47 * \sim$ & -0.09 & $1.09 * \sim$ & $2.42 * \sim$ & $3.34 * \sim$ & $3.90^{*} \sim$ \\
\hline 4. Network $1 / 2$ mile & $-5.22 * \sim$ & $-0.64^{*}$ & -0.43 & -- & 0.27 & $1.44 * \sim$ & $2.32 * \sim$ & $3.96 * \sim$ & 0.4 & $1.57^{*} \sim$ & $2.90^{*} \sim$ & $3.82 * \sim$ & $4.38 * \sim$ \\
\hline 5. Network 1 mile & $-4.91 * \sim$ & -0.33 & -0.12 & 0.31 & -- & $1.18^{*} \sim$ & $2.05 * \sim$ & $3.69 * \sim$ & 0.13 & $1.31^{*} \sim$ & $2.64 * \sim$ & $3.55^{*} \sim$ & $4.11^{*} \sim$ \\
\hline 6. Network 2 mile & $-4.16^{*} \sim$ & 0.42 & $0.63^{*}$ & $1.06 * \sim$ & $0.75^{*}$ & -- & $0.87^{*}$ & $2.51 * \sim$ & $-1.05^{*} \sim$ & 0.13 & $1.46^{*} \sim$ & $2.38 * \sim$ & $2.93 * \sim$ \\
\hline 7. Network 3 mile & $-3.21 * \sim$ & $1.38 * \sim$ & $1.59 * \sim$ & $2.02 * \sim$ & $1.71 * \sim$ & $0.96^{*}$ & -- & $1.64^{*} \sim$ & $-1.92 * \sim$ & $-0.75^{*}$ & 0.58 & $1.50 * \sim$ & $2.06 * \sim$ \\
\hline 8. Network 5 mile & $-1.51^{*} \sim$ & $3.07 * \sim$ & $3.28 * \sim$ & $3.71 * \sim$ & $3.40^{*} \sim$ & $2.65^{*} \sim$ & $1.69 * \sim$ & -- & $-3.56^{*} \sim$ & $-2.38 * \sim$ & $-1.05 *$ & -0.14 & 0.42 \\
\hline 9. Euclidean 1/2 mile & $-4.78 * \sim$ & -0.20 & 0.01 & 0.44 & 0.13 & $-0.62 *$ & $-1.58 * \sim$ & $-3.27 * \sim$ & -- & $1.8^{*} \sim$ & $2.51 * \sim$ & $3.43^{*} \sim$ & $3.98 * \sim$ \\
\hline 10. Euclidean 1 mile & $-4.18 * \sim$ & 0.40 & $0.61^{*}$ & $1.04 * \sim$ & $0.73^{*}$ & -0.02 & $-0.98 * \sim$ & $-2.67^{*} \sim$ & $0.60^{*}$ & -- & $1.33 * \sim$ & $2.25^{*} \sim$ & $2.81^{*} \sim$ \\
\hline 11. Euclidean 2 mile & $-3.19 * \sim$ & $1.39 * \sim$ & $1.60 * \sim$ & $2.03^{*} \sim$ & $1.72 * \sim$ & $0.97^{*}$ & 0.01 & $-1.68 * \sim$ & $1.59 * \sim$ & $0.99 * \sim$ & -- & $0.92^{*}$ & $1.47^{*} \sim$ \\
\hline 12. Euclidean 3 mile & $-1.95^{*} \sim$ & $2.63 * \sim$ & $2.84^{*} \sim$ & $3.27 *$ & $2.96^{*} \sim$ & $2.21^{*} \sim$ & $1.26^{*} \sim$ & -0.44 & $2.83^{*} \sim$ & $2.23^{*} \sim$ & $1.24 * \sim$ & -- & 0.56 \\
\hline 13. Euclidean 5 mile & $-0.77^{*}$ & $3.81 * \sim$ & $4.02 * \sim$ & $4.45^{*} \sim$ & $4.14^{*} \sim$ & $3.39 * \sim$ & $2.43^{*} \sim$ & $-0.74^{*}$ & $4.01 * \sim$ & $3.41^{*} \sim$ & $2.42 * \sim$ & $1.18 * \sim$ & -- \\
\hline
\end{tabular}

*Unadjusted $\mathrm{P}<0.05 ; \sim$ Bonferroni corrected $\mathrm{P}<0.05$. Note: Network and Euclidean buffer sizes are 0.5 mile/0.8 km, 1 mile/1.6 km, 2 mile/3.2 km, 3 mile/4.8 km, and 5 mile/8 km. 
block group area varies widely based on location of those block groups in urban areas, urban clusters, and rural areas with relatively larger boundaries in rural areas. For example, in our sample, the mean size of block groups within urban areas was 0.8 square miles/2.1 square $\mathrm{km}$, those in urban clusters was 1.3 square miles/3.4 square $\mathrm{km}$, and those in rural areas was 16.3 square miles/42.2 square $\mathrm{km}$. Additionally, this means that a neighborhood buffer of a given size encompasses fewer block groups in rural areas than in urban areas. In our sample, 5 mile/8 $\mathrm{km}$ Euclidean buffers located in rural areas intersected with a mean of 17.8 unique block groups whereas those in urban clusters intersected with a mean of 30.8 unique block groups, and those in urbanized areas intersected with 57.9. Thus in rural areas, disperse exposures, such as neighborhood deprivation, which are aggregated across block groups, would be expected to change more slowly as geographic boundaries expand or change shape from Euclidean to network buffers. These findings highlight the need for researchers to carefully consider scale of the neighborhood unit, especially in rural areas.

Importantly, disperse exposures (such as neighborhood deprivation) are very different from point exposures (such as parks or grocery stores) as point exposures can be counted within a certain neighborhood geography. Point exposures are likely more sensitive to different neighborhood geography types and sizes. For example, most point exposures have to be reached by road networks and thus using Euclidean or administrative buffers to capture the number of exposures may lead to biased estimates. Thus non differences that were found for rural areas and in some post-hoc comparisons in urbanized areas and urban clusters may be specific to neighborhood deprivation or to similar disperse exposures. Point exposure may be more sensitive to neighborhood definition even in rural areas. Also, given research showing how far some rural residents have to travel to access amenities like grocery stores (Thatcher et al., 2017) and for health care (Yaemsiri et al., 2019), buffer sizes many need to be even larger to even have any exposure at all. This may also suggest why there is no significant difference in deprivation across neighborhood definitions in rural settings.

A close examination of urbanized areas, shows that median levels of deprivation appear to be lower when neighborhood buffers are larger and higher at smaller neighborhood geographies. This likely reflects that the high deprivation neighborhoods in which participants from this sample live are smaller and more densely populated geographies which border on more advantaged neighborhoods. This pattern of racial and economic segregation in urban areas may in part be a direct result of historic disinvestment in certain neighborhoods through policies of institutional racism, such as redlining (Kramer, 2018; Mitchell and Franco, 2018). In post-hoc comparisons of various neighborhood definitions, we also generally see that smaller $(0.5 \mathrm{mile} / 0.8 \mathrm{~km}, 1 \mathrm{mile} / 1.6 \mathrm{~km}$, and some 2 mile $/ 3.2 \mathrm{~km}$ ) neighborhood buffers largely show no differences in comparisons with each other. Similarly, these smaller buffers do not show differences from census tracts or block groups, which would also tend to be geographically smaller in these more densely populated urbanized areas.

In urban clusters, the post-hoc comparisons revealed a less clear pattern. Null differences were found both among some of the smaller neighborhood definitions as well as among most of the larger 3 and 5 neighborhood definitions. In comparing administrative buffers to person-centric buffers, for the most part, school districts did not differ from the larger 3 mile $/ 4.8 \mathrm{~km}$ and 5 mile $/ 8 \mathrm{~km}$ buffers and block groups were similar to the smaller $0.5 \mathrm{mile} / 0.8$ $\mathrm{km}$ and $1 \mathrm{mile} / 1.6 \mathrm{~km}$ buffers. Census tracts, a commonly used neighborhood definition in developmental research, differed from all neighborhood definitions except for 1 mile/1.6 $\mathrm{km}$ and 2 mile/3.2 km Euclidean and 3 mile $/ 4.8 \mathrm{~km}$ network buffers.

\section{Implications and future directions}

These findings have several implications for researchers seeking to understand the role of neighborhood exposures in children's health and development. In rural areas, our results suggest that neighborhood definition and scale largely may not impact measured exposure to neighborhood deprivation. Still, however, we urge researchers to choose a theoretically relevant neighborhood definition when aiming to answer questions related to child health and development and argue that an ego centric neighborhood definition will likely be more theoretically relevant for children's lived experiences than utilizing administrative boundaries. For example, ego centric buffers help to overcome issues related to spatial misclassification and edge effects that are present when researchers rely on administrative boundaries. Additionally, larger rural areas may be too homogenous with respect to NDI calculations and further investigation into how to best measure NDIs is warranted, approaches such as dasymetric mapping as well as utilizing remotely sensed ancillary data have been recommended (see e.g., Baud et al., 2010). In urban areas, it is clear that researchers' choice of neighborhood definition and scale will impact the quantification of children's exposure to neighborhood deprivation and thus will likely in turn have impacts on the extent to which neighborhood deprivation is found to impact children's health and development. A growing body of work has demonstrated that relatively small, ego centric buffers may be the most relevant for urban areas (for a review see: Duncan et al., 2018). We also note the policy implications of this research, including recognizing that the most theoretically appropriate neighborhood definition may not be a policy relevant unit

Future work on child health and development thus would greatly benefit from a characterization of neighborhood context that is made on theoretical grounds rather than solely on data availability (Duncan et al., 2014). For researchers who are focused on precise measurement of developmental outcomes, this may be particularly difficult as measurement of neighborhood context may be a post-hoc addition to a study which is conducted with few resources and as privacy concerns may limit the ability to collect actual address data. The FLP data thus present a unique opportunity as the study contains rich longitudinal data on family processes and children's outcomes from birth through young adulthood including detailed geographical data. By providing descriptive information on neighborhood deprivation across multiple administrative and ego-centric neighborhood definitions, this study opens the door for more precise examination of the ways in which children's exposure to neighborhood deprivation predicts later outcomes across multiple domains of health and development. In seeking to answer these questions, future studies would greatly benefit from including multiple neighborhood definitions in order to understand what zones and sizes of neighborhood geography are most predictive of children's outcomes and to act as sensitivity checks on the role of the neighborhood geography which was chosen because of its theoretical relevance. Furthermore, in investigating the roles of different zones and sizes of neighborhood geography, future research is needed to examine how current and historical aspects of structural racism including investments and disinvestments in neighborhoods have shaped patterns of neighborhood deprivation across urban and rural areas. 


\section{Limitations}

This study is geographically limited by its focus on areas of high rural poverty in North Carolina and Pennsylvania. Thus the results showing how neighborhood deprivation differs based on choice of neighborhood definition may be specific to these areas. Furthermore, given the wide variation in the landscape of rural settings, it may be that in other rural areas, like the Southwest, measured exposure to neighborhood deprivation is more sensitive to the neighborhood definition. However, the FLP counties do capture some of this rural variation given the relatively mountainous terrain in Pennsylvania impacting settlement patterns, which is not found in this part of North Carolina (Vernon-Feagans et al., 2013). Additionally, as discussed above, it is likely that the measurement of other neighborhood characteristics not examined in this study, could be differentially sensitive to the type and scale of neighborhood buffers examined here. This may be particularly true when counting point exposures in a neighborhood such as the number of grocery stores. Future work defining rural neighborhoods may consider even larger buffers perhaps based on the distance needed to travel to obtain goods and services. Furthermore, future work examining the prediction of neighborhood deprivation to indicators of child health and development may demonstrate differences in the neighborhood definitions that are most strongly related to different outcomes.

\section{Conclusions}

Measuring neighborhood deprivation in the neighborhoods in which children live is an important step in understanding how neighborhood socioeconomic context affects child health and development. To advance this goal, this study examined the ways in which measured exposure to neighborhood deprivation differs across various definitions of children's neighborhoods in the FLP. Overall, results suggest that measured exposure to neighborhood deprivation is more sensitive to differences in neighborhood definition in urban settings than in rural settings. In describing how measured exposure to neighborhood deprivation differs across neighborhood definitions, this study informs decision making about defining neighborhoods in non-urban areas and opens the door for others to use FLP data to more precisely examine the ways in which children's exposure to neighborhood deprivation predicts later health and development.

\section{References}

Baud I, Kuffer M, Pfeffer K, Sliuzas R, Karuppannan S, 2010. Understanding heterogeneity in metropolitan India: The added value of remote sensing data for analyzing sub-standard residential areas. Int J Appl Earth Obs Geoinf 12:359-74.

Beyer HL, 2012. Geo-spatial modelling environment [Computer software] (Version 0.7.2.* RC2). Available from: http://www. spatialecology.com/gme/

Cabrera-Barona P, Wei CZ, Hagenlocher M, 2016. Multiscale evaluation of an urban deprivation index: Implications for quality of life and healthcare accessibility planning. Appl Geogr 70:1-10.

Chambers T, Pearson AL, Kawachi I, Rzotkiewicz Z, Stanley J, Smith M, Barr M, Ni Mhurchu C, Signal L, 2017. Kids in space: Measuring children's residential neighborhoods and other destinations using activity space GPS and wearable camera data. Soc Sci Med 193:41-50.

Crawford TW, Jilcott Pitts SB, McGuirt JT, Keyserling TC, Ammerman AS, 2014. Conceptualizing and comparing neighborhood and activity space measures for food environment research. Health Place 30:215-25.

De Marco A, De Marco M, 2010. Conceptualization and measurement of the neighborhood in rural settings: a systematic review of the literature. J Community Psychol 38:99-114.

Diez Roux AV, Mair C, 2010. Neighborhoods and health. Ann N Y Acad Sci 1186:125-45.

Dumedah G, Schuurman N, Yang WH, 2008. Minimizing effects of scale distortion for spatially grouped census data using rough sets. J Geogr Syst 10:47-69.

Duncan DT, Kawachi I, Subramanian SV, Aldstadt J, Melly SJ, Williams DR, 2014. Examination of how neighborhood definition influences measurements of youths' access to tobacco retailers: A methodological note on spatial misclassification. Am J Epidemiol 179:373-81.

Duncan DT, Regan SD, Chaix B, 2018. Operationalizing neighborhood definitions in health research: spatial misclassification and other issues. In: D.T. Duncan and I. Kawachi (Eds.), Neighborhoods and health. Oxford University Press, Oxford, UK, pp. 19-56.

Environmental Systems Research Institute (ESRI), 2019. ArcGIS Release 10.7.1 Redlands, CA, USA.

Finegood ED, Rarick JRD, Blair C, Family Life Project Investigators, 2017. Exploring longitudinal associations between neighborhood disadvantage and cortisol levels in early childhood. Dev Psychopathol 29:1649-62.

Fortney J, Rost K, Warren J, 2000. Comparing alternative methods of measuring geographic access to health services. Health Serv Outcomes Res Methodol 1:173-84.

Fowler CS, Jensen L, 2020. Bridging the gap between geographic concept and the data we have: The case of labor markets in the USA. Environ Plan A 0308518X20906154. [Epub ahead of print].

Hobbs M, Griffiths C, Green MA, Jordan H, Saunders J, McKenna J, 2018. Neighborhood typologies and associations with body mass index and obesity: a cross-sectional study. Prev Med 111:351-7.

Hotchkiss M, Phelan J, 2017. Uses of Census Bureau data in federal funds distribution. Available from: https://www2. census.gov/programs-surveys/decennial/2020/ programmanagement/working-papers/Uses-of-Census-Bureau-Data-inFederal-Funds-Distribution.pdf

Kramer MR, 2018. Residential segregation and health, neighborhoods and health. Oxford University Press, Oxford, UK, pp. 321-356.

Kwan MP, 2013. Beyond space (as we knew it): toward temporally integrated geographies of segregation, health, and accessibility: space-time integration in geography and GIScience. Ann Am Assoc Georgr 103:1078-86.

Leventhal T, 2018. Neighborhood context and children's development: when do neighborhoods matter most? Child Dev Perspect 12:258-63.

Lian M, Struthers J, Liu Y, 2016. Statistical assessment of neighborhood socioeconomic deprivation environment in spatial epidemiologic studies. Open J Stat 6:436-42.

Lin VK, Madill R, 2019. Incorporating spatial analyses into early care and education research. OPRE Research Brief \#2019-88. 
Washington, D.C.: Office of Planning, Research, and Evaluation, Administration for Children and Families. Available from: https://files.eric.ed.gov/fulltext/ED602065.pdf

Malik R, Hamm K, 2017. Mapping America's child care deserts. Available from: https://www.americanprogress.org/ issues/early-childhood/reports/2017/08/30/437988/mappingamericas-child-care-deserts/

Matthews SA, 2011. Spatial polygamy and the heterogeneity of place: studying people and place via egocentric methods. Communities, neighborhoods, and health. Springer, Berlin, Germany, pp. 35-55.

Messer LC, Laraia BA, Kaufman JS, Eyster J, Holzman C, Culhane J, Elo I, Burke JG, O'Campo P, 2006. The development of a standardized neighborhood deprivation index. $\mathrm{J}$ Urban Health 83:1041-62.

Minh A, Muhajarine N, Janus M, Brownell M, Guhn M, 2017. A review of neighborhood effects and early child development: How, where, and for whom, do neighborhoods matter? Health Place 46:155-74.

Mitchell B, Franco J, 2018. HOLC "Redlining" maps: The persistent structure of segregation and economic inequality; Washington, DC, USA. Available from: https://ncrc.org/wpcontent/uploads/dlm_uploads/2018/02/NCRC-ResearchHOLC-10.pdf

O'Campo, 2003. Invited commentary: advancing theory and methods for multilevel models of residential neighborhoods and health. Am J Epidemiol 157:9-13.

Östh J, Clark WA, Malmberg B, 2015. Measuring the scale of segregation using k-nearest neighbor aggregates. Geogr Anal 47:34-49.

Perchoux C, Chaix B, Brondeel R, Kestens Y, 2016. Residential buffer, perceived neighborhood, and individual activity space: New refinements in the definition of exposure areas - The RECORD cohort study. Health Place 40:116-22.

$\mathrm{R}$ Core Team, 2017. A language and environment for statistical computing. R Foundation for Statistical. Vienna, Austria. Available from: https://www.r-project.org/

Richards MP, 2014. The gerrymandering of school attendance zones and the segregation of public schools: A geospatial analysis. Am J Educ Res 51:1119-57.

Ripley BD, 1981. Spatial statistics. Wiley, New York, NY, USA.

Sadler RC, Clark AF, Wilk P, O'Connor C, Gilliland JA, 2016. Using GPS and activity tracking to reveal the influence of adolescents' food environment exposure on junk food purchasing. Can J Public Health 107:eS14-20.

Schuurman N, Bell N, Dunn JR, Oliver L, 2007. Deprivation indices, population health and geography: an evaluation of the spatial effectiveness of indices at multiple scales. J Urban Health 84:591-603.

Sluiter R, Tolsma J, Scheepers P, 2015. At which geographic scale does ethnic diversity affect intra-neighborhood social capital? Soc Sci Res 54:80-95.

Stewart O, 2011. Findings from research on active transportation to school and implications for safe routes to school programs. J Plan Lit 26:127-50.

Thatcher E, Johnson C, Zenk SN, Kulbok P, 2017. Retail food store access in rural Appalachia: A mixed methods study. Public Health Nurs 34:245-55.

US Census Bureau, April 2009. Design and methodology: American Community Survey. Available from: https://www2.census.gov/programssurveys/acs/methodology/design_and_methodology/acs_design_methodology_previous.pdf

US Census Bureau, February 2020. Urban and rural. Available from: https://www.census.gov/programs-surveys/geography/ guidance/geo-areas/urban-rural.html

Vallée J, Shareck M, 2014. Re: "Examination of how neighborhood definition influences measurements of youths' access to tobacco retailers: A methodological note on spatial misclassification". Am J Epidemiol 179:660-1.

Vernon-Feagans L, Cox M, Family Life Project Key Investigators, 2013. The Family Life Project: an epidemiological and developmental study of young children living in poor rural communities. Monogr Soc Res Child Dev 78:1-150.

Wan C, Su SL, 2016. Neighborhood housing deprivation and public health: Theoretical linkage, empirical evidence, and implications for urban planning. Habitat Int 57:11-23.

Willoughby M, Burchinal M, Garrett-Peters P, Mills-Koonce R, Vernon-Feagans L, Cox M, 2013. The Family Life Project: An epidemiological and developmental study of young children living in poor rural communities: II. Recruitment of the Family Life Project sample. Monogr Soc Res Child Dev 78:24-35.

$\mathrm{Xu} \mathrm{H,} \mathrm{Logan} \mathrm{JR,} \mathrm{Short} \mathrm{SE,} \mathrm{2014.} \mathrm{Integrating} \mathrm{space} \mathrm{with} \mathrm{place} \mathrm{in}$ health research: a multilevel spatial investigation using child mortality in 1880 Newark, New Jersey. Demography 51:811-34.

Yaemsiri S, Alfier JM, Moy E, Rossen LM, Bastian B, Bolin J, Ferdinand AO, Callaghan T, Heron M, 2019. Healthy people 2020: Rural areas lag in achieving targets for major causes of death. Health Aff 38:2027-31.

Zenk SN, Schulz AJ, Matthews SA, Odoms-Young A, Wilbur J, Wegrzyn L, Gibbs K, Braunschweig C, Stokes C, 2011. Activity space environment and dietary and physical activity behaviors: A pilot study. Health Place 17:1150-61. 